\title{
Evaluation of Cardiac Thrombus Stability based on Contrast-Enhanced Echocardiography凶Research Protocol and Preliminary Results
}

\section{Ying Li}

Department of Ultrasound, Shengjing Hospital of China Medical University

Xin Wang ( $\square$ sisterxiaoxin@163.com )

Department of Ultrasound, Shengjing Hospital of China Medical University

\section{Weidong Ren}

Department of Ultrasound, Shengjing Hospital of China Medical University

\section{Yangjie Xiao}

Department of Ultrasound, Shengjing Hospital of China Medical University

\section{Xiaona Yu}

Department of Ultrasound, Shengjing Hospital of China Medical University

\section{Xueying Tan}

Department of Ultrasound, Shengjing Hospital of China Medical University

\section{Research Article}

Keywords: Cardiac, CEUS, SWE, TTE

Posted Date: February 24th, 2021

DOI: https://doi.org/10.21203/rs.3.rs-228409/v1

License: (1) This work is licensed under a Creative Commons Attribution 4.0 International License. Read Full License 


\section{Title Page}

Title: Evaluation of Cardiac Thrombus Stability based on Contrast-Enhanced Echocardiography: Research Protocol and Preliminary Results

Author: Ying Li, Xin Wang, Weidong Ren, Yangjie Xiao, Xiaona Yu, Xueying Tan

Affiliation: Department of Ultrasound, Shengjing Hospital of China Medical University, Shenyang, China

\section{Corresponding author:}

Name: Xin Wang, M.D.

Address: Department of Ultrasound, Shengjing Hospital of China Medical University, Shenyang, China (No.36, Sanhao Street, Heping District, Shenyang City, China, 110004).

Email: sisterxiaoxin@163.com

Telephone number: +86-18940258303 
Evaluation of Cardiac Thrombus Stability based on Contrast-Enhanced Echocardiography:

Research Protocol and Preliminary Results

Abstract

Objective: This study attempted to test a new scoring system for evaluating the stability of cardiac thrombi using contrast-enhanced ultrasound (CEUS).

Methods: We used human whole blood to make the thrombus model in vitro which were divided into 2 groups, the 1-hour $\left(T_{1 \mathrm{~h}}\right)$ and 7 -day $\left(T_{7 \mathrm{~d}}\right)$ group. The $\mathrm{T}_{1 \mathrm{~h}}$ group was monitored for 1 hour continuously to observe for the formation of a new thrombus on the original thrombus base. Over time, changes in CEUS images and pathology and shear wave elastography (SWE) of thrombus were observed in the $T_{1 \mathrm{~h}}$ and $\mathrm{T}_{7}$ groups. Twenty-eight adult patients diagnosed with a cardiac thrombus were selected and examined by transthoracic echocardiography (TTE) and CEUS. Thrombi were scored for substrate $\left(T_{s}\right)$ and hardness $\left(T_{h}\right)$ based on the visualized degree of contrast penetration into the thrombus. The $T_{s}$ and $T_{h}$ were statistically analyzed for thrombolytic time and the risk of embolism to other organs.

Results: In vitro, pathology revealed a decreased pore structure; further, the average Young's modulus of the thrombi over time indicated a progressive increase in hardness. Contrast-enhancing agents were able to enter a fresh, loose thrombus, but were not able to enter a chronic, stable thrombus. With increasing $T_{s}$ and $T_{h}$, thrombolytic time was prolonged, and the risk of embolism to other organs was increased.

Conclusions: Our study results suggest that this new CEUS scoring system can evaluate the hardness of a cardiac thrombus and the quality of its underlying substrate; this allows for quantitative evaluation of thrombus stability.

\section{Introduction}

Cardiac thrombus formation is a frequent complication of a variety of prevalent diseases[1, 2]. It has the potential to result in significant morbidity and mortality from cerebrovascular and peripheral vascular events[3]. In recent years, many scholars have begun to study the risk factors of thrombus shedding. Most people believe that there is a certain correlation between the time of thrombosis formation and the risk of thrombus shedding and the effect of thrombolysis[4]. In the acute stage, the unstable thrombus was less rigid and had loose adhesion with the vascular wall, and the thrombus was easy to break and fall off, but treatment results are usually good. Chronic 
thrombus was relatively stable, the hardness and toughness of thrombus also increased, the brittleness decreased, and the adhesion with vascular wall was closer, and it was not easy to fall off, and treatment results are usually poor $[5,6]$. SWE is an accurate method to determine the time of thrombosis according to the changes of thrombus hardness[7]. Therefore, the hardness of thrombus is related to the risk of falling off and the effect of thrombolysis[5]. According to the above theoretical basis, we may speculate the risk of thrombus shedding and thrombolytic effect according to the changes of the hardness of cardiac thrombus.

At present, the most commonly used method for diagnosing cardiac thrombus is TTE which often focus on their size, number, shape, activity, and location[8, 9]. To our knowledge, we lack the means to judge the hardness which is related to stability of cardiac thrombus at present. And the TTE image quality is suboptimal in some patients, which leads to relatively low accuracy and reproducibility[10, 11]. It has been demonstrated the introduction of a contrast agent during TTE improves image quality and display the blood supply of the tissue by observing the microbubbles of contrast medium moving with the red blood cells[12, 13]. There are only a few studies on the application of CEUS in the diagnosis of cardiac thrombosis. According to the previous study, unlike solid tumors, no contrast agent can enter the thrombus without blood supply during CEUS[11, 14, 15].

However, it is well known that in physics, all molecules have the physical characteristics of mechanical dispersion[16]. This is due to the separation and reorganization of streamline due to the interaction with the solid surface, which is affected by the pore structure of the medium under consideration[17]. There is no contrast medium in the hard and chronic thrombus, but does the soft and fresh thrombus have the pore structure through which the contrast agent can pass? Can we take advantage of the mechanical diffusion properties of contrast media to judge the hardness of thrombus?

To confirm the hypothesis, we designed the first part of the in vitro experiment attempted to observe that the change of thrombus with the extension of formation time, and if the contrast agent could enter fresh thrombus or not. Then we studied 28 patients with cardiac thrombi to further evaluate the clinical stability of cardiac thrombosis based on the performance of CEUS.

\section{Materials and methods}

\section{Study participants}


The thrombus model in vitro was established using a $10.0 \mathrm{~mL}$ portion of whole venous blood collected from healthy volunteers and placed in a $20 \mathrm{~mL}$ syringe in a $37^{\circ} \mathrm{C}$ bath box (10 syringes in total)[18]. According to different thrombosis times, the blood samples were divided into two groups, the 1-hour $\left(T_{1 h}\right)$ and 7-day $\left(T_{7 d}\right)$ group, with 5 samples in each group. The thrombus was analyzed using 2-dimensional TTE and CEUS and SWE.

From May 1, 2016 to June 29, 2019, 28 consecutive patients who had been diagnosed with intracardiac thrombus by echocardiography were recruited for this study. The diagnoses of all of the patients were further confirmed by clinical diagnosis. This study was approved by the ethics committee of Shengjing Hospital affiliated with China Medical University, and written consent was obtained from all patients before the study. All procedures were performed in accordance with the 1964 Declaration of Helsinki and its later amendments or comparable ethical standards. Twenty-eight patients met the following diagnostic criteria: (1) recent thrombolytic therapy, (2) echocardiography was performed every three days, and (3) cardiac masses decreased in size or fully resolved.

\section{Ultrasonography}

The Philip EPIC7 (with an L12-3 transducer and 3-12 MHz transmission frequency) ultrasound system was used to perform 2-dimensional ultrasound imaging and CEUS imaging of the thrombus in vitro. A Supersonic Aixplorer (with an L15-4 transducer and 4-15 MHz transmission frequency) ultrasound system was used to analyze the SWE of thrombus in vitro.

A Philip iE elite (with an S5-1 transducer and 1-5 MHz transmission frequency) ultrasound system was used to examine the patients with cardiac thrombosis. Images were analyzed with the image analysis software QLAB, version 10.8. Approximately $3 \mathrm{~mL}$ of the contrast agent perfluoropropane human albumin microsphere injection fluid (Hunan Kangrun Pharmaceutical Company, China) was used. It was composed of $1 \%$ human albumin-coated perfluoropropane albumin microspheres.

\section{Image acquisition}

The $T_{1 \mathrm{~h}}$ group $T_{7 d}$ group in vitro were observed using 2-dimensional and SWE and CEUS. The $\mathrm{T}_{1 \mathrm{~h}}$ group was monitored by ultrasound for 1 hour continuously to observe for the formation of a new thrombus on the original thrombus base. After 1 hour continuously observe, CEUS was performed in vitro as follows: (1) the contrast agent was injected into the normal saline bag, (2) the 
syringe containing the thrombus was connected to the saline bag, and (3) the normal saline containing the mixed contrast agent was slowly and continuously aspirated. The evaluation of thrombus SWE was as follows: (1) the thrombus form was traced, (2) the average Young's modulus value (the sum of the minimum and maximum Young's modulus values [unit: $\mathrm{kPa}$ ] of the thrombus within the Q-box area according to the system was automatically calculated, and (3) the average Young's modulus value was selected for further statistics. After calculating the Young's modulus three times, the mean value was used for statistics. The $T_{7 d}$ group was monitored by ultrasound to observe for the formation of chronic thrombi on the original thrombus base. The method is the same as before.

Patients took the left lateral decubitus position, and electrocardiography (ECG) was recorded simultaneously. A total of 3 to 5 cardiac cycles of the left ventricular short-axis view was acquired (the mitral valve, papillary muscle, and apical horizontal section separately), in addition to the left ventricle long-axis 3-chamber view, the apical 2-chamber view, and the apical 4-chamber view, as well as other nonstandard sections. The location, attachment point, substrate, shape, size, internal echo, activity, and hemodynamics of the intracardiac thrombus were observed. Long and short diameters were measured at the largest section of the thrombus. Subsequently, all patients were examined by CEUS. The left ventricular opacification (LVO) mode was started, and the contrast agent was gently mixed. After reaching room temperature, $1 \mathrm{~mL}$ of contrast agent was injected from the extremities. The previously mentioned basic biologic heart filling and lesion characteristics were observed. The long and short diameter and the area of the thrombus were measured at the largest section. The contrast agent entering around and at the base of the thrombus was observed and recorded. After that, the patients were examined by echocardiography every 3 days. Images were stored on hard disk for off-line analysis.

\section{Image analysis}

The size, echo, activity, time-dependent changes, contrast agent entry, and elastography of thrombus in vitro were recorded. For the semi-quantitative analysis, the degree of contrast enhancement of the cardiac thrombus was divided into 4 grades by visual observation.

The substrate condition of the cardiac thrombus is defined as $T_{s}$ :

- 1 point: no or less than $1 / 2$ of the substrate could be seen with the mixing of spot-like $(<3 \mathrm{~mm})$ contrast agent 
- 2 points: more than $1 / 2$ of the substrate could be seen with the mixing of crack-like contrast agent

- 3 points: a wide gap ( $>3 \mathrm{~mm}$ ) of ultrasound contrast agent could be seen in most of the substrate

- 4 points: no or thin substrate

The hardness of the cardiac thrombus is defined as $T_{h}$ :

- 1 point: no contrast agent was found on the surface of the thrombus

- 2 points: a small amount of dot-like contrast agent was seen on the surface of the thrombus

- 3 points: strip-like contrast agent was seen on the surface of the thrombus

- 4 points: a large amount of the contrast agent entered the surface of the thrombus

- The standard of CEUS scoring system is shown in Figure 1. The total CEUS score is equal to $T_{s}$ plus $T_{h}$.

\section{Inter-observer and intra-observer reproducibility}

To assess the reproducibility of the quantitative measurements, $T_{s}$ and $T_{h}$ were measured in 8 patients by 2 independent observers. Observers $A$ and $B$ (both observes had the same qualifications) measured the patients once on the same day; observer A measured the same sample one more time after 1 week. Inter-observer and intra-observer variability were calculated as the percentage of the absolute difference between the 2 measurements divided by the mean value of the measurements.

\section{The thrombolytic time, and other embolic sites}

Twenty-eight patients received thrombolytic therapy and underwent TTE review every three days until the cardiac thrombus disappeared and the time was recorded as the thrombolytic time. When the patient was diagnosed with cardiac thrombosis for the first time, auxiliary examination was performed on the symptom related organs to confirm whether the patient had other organ infarction. For example, vomit for abdominal vascular ultrasound, dizziness or headache for head CT scan, leg pain for lower extremity vascular ultrasound. We recorded the presence and absence of other 
embolism organs as 1 and 0.

\section{Statistical analysis}

Statistical software SPSS, version 23.0, was used to analyze the data. The measurement data conformed to a normal distribution and the variance was expressed as mean \pm standard deviation. The two groups of parameters were compared by independent sample $t$ test. Non-normal distributions were represented by median and interquartile range, and the two sets of parameters were compared by independent sample nonparametric tests. The count data were expressed as the rate. The $X^{2}$ test or Fisher exact test was used, and the difference was statistically significant at $P<0.05$ (2-sided). The correlation between CEUS score and thrombolysis time and other organ embolism was analyzed by single factor correlation analysis.

\section{Pathology}

The thrombus in vitro was fixed with $4 \%$ formaldehyde, dehydrated, embedded, stained with hematoxylin eosin, and dehydrated again. The thrombus was observed under the light microscope.

\section{Results}

\section{Ultrasonic features of thrombus in vitro}

The $T_{1 \mathrm{~h}}$ group was observed for 1 hour continuously. The surface of the thrombus in $T_{1 \mathrm{~h}}$ group was hypoechoic (Figure 2A), the ultrasound contrast agent can enter the freshly formed part of the thrombus surface (Figure 2D), which makes the contrast morphology inconsistent with the 2-dimensional morphology. Two hours after the formation of the thrombus, the volume of the thrombus increased continuously compared with the $T_{1 \mathrm{~h}}$ thrombus group (mean \pm SD: $16.32 \pm$ 5.34 vs $28.43 \pm 8.43 \mathrm{~mL}, P<0.05)$. The echo of the surface of the thrombus was enhanced and it was hyperechoic (Figure 2B). A small thrombus with obvious mobility on the surface of the thrombus can be seen (Figure 2E), which is not shown in the two-dimensional image. The inside of the thrombus in $T_{7 d}$ group was uniformly hypoechoic, and the echo of the surface was slightly stronger (Figure $2 \mathrm{C}$ ). No ultrasound contrast agent entered the surface of the thrombus in $\mathrm{T}_{7 \mathrm{~d}}$ group ((Figure 2F).

SWE showed that compared with the fresh thrombus group, the Young's modulus of the $T_{1 \mathrm{~h}}$ and $T_{7 d}$ groups was higher, and the Young's modulus of the $T_{7 d}$ group was higher than that of the $\mathrm{T}_{\text {1h }}$ (mean $\pm \mathrm{SD}: 3.85 \pm 2.24 \mathrm{kPa}$ vs $9.85 \pm 3.24 \mathrm{kPa}$ vs $28.58 \pm 4.23 \mathrm{kPa}, P<0.05$ in the fresh, $\mathrm{T}_{1 \mathrm{~h}}$ 
and $T_{7 d}$ groups, respectively ). The SWE images are shown in Figure 3.

\section{Pathology of thrombus in vitro}

The fresh thrombus had the loosest structure and the least cell aggregation. One hour later, the structure of the thrombus fiber grid was loose and the cell space was large. On the seventh day, the structure of the red blood cells and the fiber network was uniform, and the intercellular space was small (Figure 4).

\section{Population characteristics}

Characteristics of the patient population are shown in Table 1. The average age of the 28 patients was (mean age \pm SD) $52.6 \pm 16.8$ years old, including 17 male and 11 female, and no adverse drug reactions were observed. Men were significantly more affected than women $(P<$ 0.05); 6 types of cardiac disease were involved in this study. Among the 28 cases, $26(92.86 \%)$ had cardiac disease, 4 (14.29\%) had rheumatic valvular disease, 4 (14.29\%) had coronary heart disease, 4 (14.29\%) had simple atrial fibrillation, $6(21.43 \%)$ had hypertension, 7 (25\%) had cardiomyopathy, 1 (3.57\%) had infective endocarditis, 6 patients had 2 or more types of heart disease $(21.43 \%)$, and 2 patients $(7.14 \%)$ had non-cardiac disease. The thrombolytic time ranged from three days to 90 days. $10(35.71 \%)$ had the other embolic sites including brain, kidney, abdominal aorta, posterior tibial artery.

\section{Cardiac thrombus echocardiographic features}

The overall distribution of the cardiac thrombus was as follows: most of the 28 cases occurred with a single site, and a few occurred in two or more sites simultaneously. $22(78.57 \%)$ of the cases had a single embolus, 6 (21.43\%) had multiple emboli, 5 (17.85\%) had 2 emboli, and 1 (3.57\%) had 3 emboli. A total of 35 emboli were found. There were 2 emboli in the left atrial appendage (5.71\%), 10 in the left atria (28.57\%), 22 in the left ventricle $(62.86 \%)$, and 1 found in the right ventricle $(2.86 \%)$.

Conventional TTE showed the cardiac thrombus as a mass-like echo in the heart, and the shapes could be irregular, round, crescent or wedge-shaped. Most of the thrombi had a wide base, and a few had no base. Most of the thrombi had no obvious activity or deformation. The thrombi without a base showed obvious activity and slight deformation. The echoes of the thrombi were either hypoechoic, hyperechoic, or had a mixed echo. The thrombus size ranged from $16 \times 10 \mathrm{~mm}$ to $53 \times 43 \mathrm{~mm}$. 
The CEUS revealed that the contrast agent could enter the surface and base of a partial thrombus. The contrast images of some thrombi were inconsistent with the 2-dimensional images. The contrast images were scored by a set contrast-enhanced scoring system. Table 2 showed the specific score.

Through a case, we showed the changes in the CEUS appearance of thrombus after thrombolytic therapy and the corresponding scores. A woman aged 18 years with infective endocarditis of 11 years' duration was diagnosed with cerebral, renal, and lower extremity arterial embolism. TTE showed three thrombi in the left ventricular cavity (Figure 5A and 5B). The CEUS showed the contrast agent entered the surface of the three thrombi. The morphology was inconsistent with the 2-dimensional morphology. There was no substrate noted in thrombus 1 and thrombus 2 (Figure $5 \mathrm{C}$ ). The CEUS score was $T_{s} 4+T_{h} 4$. The thrombus 1 and thrombus 2 disappeared with 6 days of thrombolytic therapy. Wide gap accumulation of contrast agent can be seen in most of the base of the thrombus 3 (Figure 5D). The CEUS score was $T_{s} 3+T_{h} 4$. After 9 days of thrombolytic treatment, the thrombus 3 showed that the contrast agent entered in the form of spots, and the CEUS score was $T_{s} 3+T_{h} 2$ (Figure 5E). The thrombus 3 resolved 18 days after thrombolytic therapy.

The relationship between the thrombus CEUS score, the thrombolytic time, and other embolic sites

According to the statistics of the CEUS score, thrombolysis time, and embolism of other organs outside the heart, it was found that the $T_{s}, T_{h}$ and the total score were correlated with thrombolytic time. It was hypothesized that with an increase in the $T_{s}, T_{h}$, and the total score, the thrombolytic time would be prolonged (Table 3 and 5 ). Our results suggest that the $T_{s}, T_{h}$, and total score were correlated with embolism in other organs. It was also hypothesized that with an increase in $T_{s}, T_{h}$ and the total score, the risk of embolism in other organs would also increase (Table 4 and 5).

\section{Inter-observer and intra-observer reproducibility}

The inter-observer variability of the $T_{s}$ and $T_{h}$ measurements was $11.26 \pm 6.4 \%$ and $10.26 \pm$ $6.4 \%$, respectively. The intra-observer variability was $9.65 \pm 4.2 \%$ and $8.65 \pm 5.2 \%$, respectively.

\section{Discussion}

Cardiac embolic events account for up to $30 \%$ of all ischemic strokes[2]. Accurate 
decision-making in the evaluation of cardiac thrombi stability is critical to guide therapy and procedure planning[1]. TTE is widely accepted as a primary screening tool in the evaluation of cardiac thrombus because it is noninvasive, convenient, and has a high specificity and sensitivity[9]. However, there are many limitations[10]. First, TTE cannot accurately judge the presence of a thrombus if the image quality is poor. Second, TTE lacked in-depth information on the stability of the thrombus. CEUS is a technology developed since the mid 1980's, which can not only improve the image quality but also display the blood supply of the tissue by observing the microbubbles of contrast medium moving with the red blood cells[19]. Therefore, we decided to observe cardiac thrombus by CEUS, and proposed a scoring system of cardiac thrombus for the first time. According to the previous study, unlike solid tumors, no contrast agent can enter the thrombus without blood supply during CEUS. However, this previous investigation ignored the unique property of the time-dependent nature of the thrombus[20]. We wondered if the loose texture of a fresh thrombus lacks contrast agent similar to an organized thrombus.

The research included an Initial in vitro study followed by a human protocol, in which we studied 28 patients with cardiac thrombi who were examined using CEUS imaging. In the first part, we observed the changes in pathology and SWE of a thrombus over time and changes to the contrast medium entering the thrombus over time through in vitro experiments; this was done to determine whether or not contrast agent entering a thrombus changes with the hardness.

SWE has been proved to be able to quantitatively evaluate tissue hardness in a noninvasive way by obtaining the Young's modulus of the corresponding part, which objectively reflects its elasticity[21]. The higher the Young's modulus, the harder the tissue[7]. We used real-time quantitative SWE to determine the change of thrombus hardness by measuring the elastic value of the thrombus in vitro at different points in time and observed the hardness changes of the thrombus over time. The results showed that compared with the fresh thrombus group, the Young's modulus of $T_{1 \mathrm{~h}}$ and $T_{7 \mathrm{~d}}$ groups were higher, and the $T_{7 \mathrm{~d}}$ group was higher than that $T_{1 \mathrm{~h}}$ group. Through the observation SWE, we found that the hardness of thrombus which is related to stability and indicates the risk of thrombus fragmentation and shedding increases as time goes by.

We also found that during formation of the thrombus, cell aggregation increased the internal portion of the thrombus changed from loose to dense in pathological observation. Through the 
observation of pathology, we can prove that the pore structure of thrombus decreased with time. Through the above pathological and hardness changes, we can conclude that as the pore structure of thrombus becomes smaller, the hardness of thrombus increases.

We observed thrombus formation continuously for 1 hour and found that contrast agent can enter the fresh thrombus. In the $T_{7 d}$ group, the contrast medium did not enter. This confirmed that contrast agents can enter fresh thrombus with large pore structure, which is a common phenomenon. Combined with the phenomenon of contrast agent entering into loose fresh thrombus indicated that the phenomenon of contrast agent entering into the space-occupying area of a thrombus not only reflected the level of angiogenesis in that area, but also reflected the pore structure associated with hardness of the space-occupying area. The phenomenon of contrast agent entry can reflect the thrombus hardness which provides a basis for us to judge the stability of the thrombus. At the same time, it is difficult to distinguish by conventional ultrasound the initial stage of thrombosis because of the low and weak echo seen in 2-dimensional sonography. However, CEUS can make a correct diagnosis.

The purpose of the second part of our study was to verify the correlation between contrast agent entry and thrombus stability. Through the course of our study, unlike thrombus formation in vitro, we discovered that the contrast medium not only enters into a cardiac thrombus, but its degree of entry also reflects the state of the thrombus substrate. In order to systematically explain CEUS imaging of cardiac thrombus formation, we first proposed a scoring system to evaluate cardiac thrombi. We scored thrombus by combining the contrast agent entry and substrate conditions. To judge the clinical significance of the new scoring system for the evaluation of thrombus stability, the $T_{s}$ and $T_{h}$ of a cardiac thrombus, the clinical thrombolysis time, and embolism to other organs were statistically analyzed.

Through the statistical analysis of the total score, thrombolytic time, and other organ embolism, we can preliminarily conclude that with an increase in $T_{s}, T_{h}$, and the total score, thrombolytic time will be prolonged and the risk of other organ embolism will increase. The higher the $T_{s}$ score, the more unstable the base is, and the higher the $T_{h}$ score, the looser the thrombus and the higher risk of local shedding but the thrombolytic effect improves. The degree of $T_{s}$ and $T_{h}$ can reflect the stability of cardiac thrombus which associate with its hardness.

According to the results of the first part of the experiment, the fresh thrombus with loose 
texture do not lack contrast agent different from a dense thrombus. The second part of our experiment confirmed that the degree of contrast media can reflect the stability of cardiac thrombus, thus indicating the prognosis of thrombus.

In the future, we can predict the prognosis of patients according to the thrombus CEUS scoring system, and further guide their treatment and care in clinical practice.

\section{Limitations}

Due to the limitations of financial cost and time, CEUS cannot be widely used in clinical practice. Therefore, we have not obtained a large sample of CEUS data regarding cardiac thrombus analysis. At the same time, thrombi in vitro cannot completely simulate the pathological changes of thrombi in vivo. In the future, we will further expand the sample size and conduct in vivo experiments in order to obtain more information in this field.

\section{Conclusions}

CEUS can detect the initial formation of thrombi that are difficult to detect with TTE. Ultrasound contrast agent can enter the fresh portion and interstitial substrate of the thrombus. The stability of the thrombus can be preliminarily judged by a CEUS scoring system, and the thrombolytic time of the thrombus and the possibility of embolism to other organs can be predicted. The new CEUS scoring system can evaluate the hardness and substrate of a thrombus, which allows for the quantitative analysis of thrombus stability.

\section{Abbreviations}

CEUS: Contrast-Enhanced Ultrasound, SWE: Shear Wave Elastography, $\mathrm{T}_{\mathrm{h}}$ : Thrombus Hardness, $T_{\mathrm{s}}$ : Thrombus Substrate, TTE: Transthoracic Echocardiography

\section{Declarations}

Acknowledgements: We thank all of the patients involved in this study. We thank Yao Xu for his voluntary blood donation for this research.

Author's contribution: YL conceived and designed the study, drafted the manuscript. XW interpreted and revised the manuscript. WDR and YJX are involved in ultrasound diagnosis. XNY and XYT analyzed and interpreted data. All authors read and approved the final manuscript.

\section{Funding: None.}

Availability of data and materials: The datasets used in the manuscript are available from the corresponding author upon reasonable request. 
Ethics approval and consent to participate: This study was approved by the ethics committee of Shengjing Hospital affiliated with China Medical University, and written consent was obtained from all patients before the study. All procedures were performed in accordance with the 1964 Declaration of Helsinki and its later amendments or comparable ethical standards.

Consent for publication: Written informed consent was obtained from all the patients for publication of this manuscript and any accompanying images. The copy of the written consent is available for review by the Editor-in-Chief of this journal.

\section{Competing interests: None.}

\section{Reference}

1. Mollazadeh R, Ostovan MA, Abdi Ardekani AR. Right cardiac thrombus in transit among patients with pulmonary thromboemboli. Clin Cardiol. 2009;32(6):E27-31.

2. Hannon N, Sheehan O, Kelly L, et al. Stroke Associated with Atrial Fibrillation Incidence and Early Outcomes in the North Dublin Population Stroke Study. Cerebrovasc Dis. 2010;29(1):43-49.

3. Sen S, Lima JAC, Oppenheimer SM. Changes in cardiac thrombus status after cerebral ischemia. Cerebrovasc Dis. 2004;17(2-3):175-181.

4. Rubin JM, Xie H, Kim K, et al. Sonographic elasticity imaging of acute and chronic deep venous thrombosis in humans. J Ultrasound Med. 2006;25(9):1179-1186.

5. Yi X, Ni C, Li M, Wu Q, Li Y. Correlation between biological characteristics of thrombus and short-term efficiency of thrombolytic therapy by acoustic radiation force impulse imaging technique. Int Angiol. 2017;36(6):574-579.

6. Takimura H, Hirano K, Muramatsu T, et al. Vascular Elastography: A Novel Method to Characterize Occluded Lower Limb Arteries Prior to Endovascular Therapy. J Endovasc Ther. 2014;21(5):654-661.

7. Yoon JH, Lee JM, Woo HS, et al. Staging of Hepatic Fibrosis: Comparison of Magnetic Resonance Elastography and Shear Wave Elastography in the Same Individuals. Korean Journal of Radiology. 2013;14(2):202-212.

8. Weinsaft JW, Kim HW, Crowley AL, et al. LV Thrombus Detection by Routine Echocardiography Insights Into Performance Characteristics Using Delayed Enhancement CMR. Jacc-Cardiovascular Imaging. 2011;4(7):702-712. 
9. Whalen H, Dako F, Patel P, et al. Role of Imaging for Suspected Cardiac Thrombus. Curr Treat Options Cardiovasc Med. 2019;21(12):81.

10. Groeneveld NS, Guglielmi V, Leeflang MMG, et al. CT angiography vs echocardiography for detection of cardiac thrombi in ischemic stroke: a systematic review and meta-analysis. J Neurol. 2020;267(6):1793-1801.

11. Mansencal N, Revault-d'Allonnes L, Pelage J-P, Farcot J-C, Lacombe P, Dubourg O. Usefulness of contrast echocardiography for assessment of intracardiac masses. Arch Cardiovasc Dis. 2009;102(3):177-183.

12. Hamilton-Craig C, Sestito A, Natale L, et al. Contrast transoesophageal echocardiography remains superior to contrast-enhanced cardiac magnetic resonance imaging for the diagnosis of patent foramen ovale. Eur $\mathrm{J}$ Echocardiogr. 2011;12(3):222-227 .

13. Saloux E, Labombarda F, Pellissier A, et al. Diagnostic Value of Three-Dimensional Contrast-Enhanced Echocardiography for Left Ventricular Volume and Ejection Fraction Measurement in Patients With Poor Acoustic Windows: A Comparison of Echocardiography and Magnetic Resonance Imaging. J Am Soc Echocardiogr. 2014;27(10):1029-1040.

14. Zhang XT, Li Y, Ren SH, et al. Isolated metastasis of hepatocellular carcinoma in the right ventricle. BMC Cardiovasc Disord. 2019;19(1):287.

15. Wang X, Li Y, Ren W, Yu X, Tan X. Clinical diagnostic value of contrast-enhanced ultrasonography in the diagnosis of cardiac masses: A pilot study. Echocardiography. 2020;37(2):231-238.

16. Grant GP, Gerhard JI. Simulating the dissolution of a complex dense nonaqueous phase liquid source zone: 2. Experimental validation of an interfacial area - based mass transfer model. Water Resources Research. 2007;43(12).

17. Wang $\mathrm{H}$, Lun Z, Lv C, et al. Measurement and Visualization of Tight Rock Exposed to CO2 Using NMR Relaxometry and MRI. Sci Rep. 2017;7:44354.

18. Bader KB, Gruber MJ, Holland CK. SHAKEN AND STIRRED: MECHANISMS OF ULTRASOUND-ENHANCED THROMBOLYSIS. Ultrasound Med Biol. 2015;41(1):187-196. 
19. Senior $R$, Becher $H$, Monaghan $M$, et al. Clinical practice of contrast echocardiography: recommendation by the European Association of Cardiovascular Imaging (EACVI) 2017. European Heart Journal-Cardiovascular Imaging. 2017;18(11):1205.

20. Morris, Timothy A. Natural history of venous thromboembolism. British Journal of Surgery. 2011;27(4):869-884.

21. Xue E, Yu Y, Lin L, Li Z, Su H. Application value of real-time shear wave elastography in differential diagnosis of testicular torsion. Med Ultrason. 2020;22(1):45-50.

\section{Figure Legend:}

Figure 1. The scoring standard of contrast-enhanced ultrasound scoring system.

A: $T_{s}$ 1: no or less than $1 / 2$ of the substrate could be seen with the mixing of spot-like $(<3 \mathrm{~mm})$ contrast agent (red arrow); $T_{h} 1$ : no contrast agent was found on the surface of the thrombus.

$B: T_{s} 2:$ more than $1 / 2$ of the substrate could be seen with the mixing of crack-like contrast agent (red arrow); $T_{h}$ 2: a small amount of dot-like contrast agent was seen on the surface of the thrombus (yellow arrow).

C: $T_{s}$ 3: a wide gap (>3 mm) of ultrasound contrast agent could be seen in most of the substrate (red arrow); $T_{h}$ 3: strip-like contrast agent was seen on the surface of the thrombus (yellow arrow). $D: T_{s}$ 4: no or narrow/thin substrate (red arrow); $T_{h} 4$ : a large amount of the contrast agent entered the surface of the thrombus (yellow arrow).

Figure 2. The two-dimensional and contrast-enhanced ultrasound appearance of the thrombus at 1 hour, 2 hours and 7 days.

A: One hour after thrombus formation, the surface of the thrombus was hypoechoic (blue arrow). B: Two hours after the formation of the thrombus, the volume of the thrombus increased, the echo of the surface of the thrombus was enhanced and it was hyperechoic (blue arrow). C: Seven days after the thrombus formed, the inside of the thrombus was uniformly hypoechoic, and the surface echo was slightly stronger (blue arrow). D: One hour after thrombus formation, the ultrasound contrast agent can enter the freshly formed part of the thrombus surface (blue arrow), which makes the contrast morphology inconsistent with the 2-dimensional morphology. E: Two hours 
after the formation of the thrombus, a small thrombus (blue arrow) with obvious mobility on the surface of the thrombus can be seen, which is not shown in the two-dimensional image. F: No ultrasound contrast agent entered the surface of the thrombus at 7 days (blue arrow).

Figure 3. Shear wave elastography images at different time periods after thrombosis.

A: fresh thrombosis; B: thrombus formed within 1 hour; C: thrombus formed over 7 days.

Figure 4. Pathologic staining results (Hematoxylin-eosin staining $\times 200)$.

A: The newly formed thrombi had the loosest texture and minimal cell aggregation. B: The fibrous network structure was loose and the cell space was large shows that 1 hour after thrombus formation. C: The structure of the red blood cells and fiber network was uniform, and the intercellular space was small on the seventh day.

Figure 5. A woman aged 18 years with infective endocarditis of 11 years' duration was diagnosed with cerebral, renal, and lower extremity arterial embolism. TTE and CEUS showed three thrombi in the left ventricular cavity. All of the thrombi resolved after thrombolytic therapy.

Figure A: TTE showed two solid, quasi-circular thrombi (thrombus 1 - blue arrow, thrombus 2 - red arrow) in the left ventricle. Figure B: TTE showed one solid, irregular thrombus (thrombus 3 yellow arrow) in the left ventricle apex. Figures C: The CEUS showed a large amount of contrast agent entered the around of the thrombus 1 (blue arrow) and thrombus 2 (red arrow). The morphology was inconsistent with the 2-dimensional morphology. There was no substrate noted in thrombus 1 and thrombus 2, the CEUS score was $T_{s} 4+T_{h} 4$. Figures $D$ : The CEUS showed a large amount of contrast agent entered the around of the thrombus 3 (yellow arrow). Wide gap accumulation of contrast agent can be seen in most of the base of the thrombus 3 , the CEUS score was $T_{s} 3+T_{h} 4$. Figures $E$ : The CEUS showed that the contrast agent entered the thrombus 3 (yellow arrow) in the form of spots after 9 days of thrombolytic treatment. The CEUS score was $T_{s} 3+T_{h} 2$. 


\section{Figures}

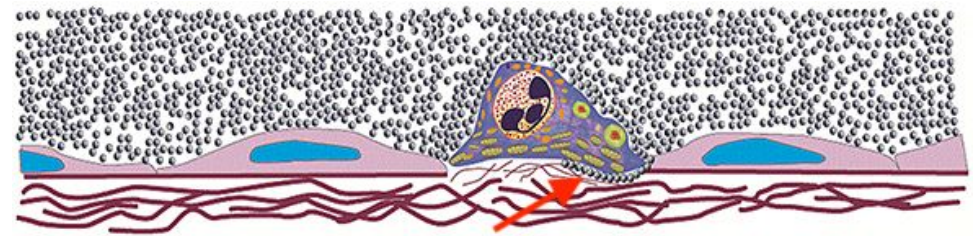

$\mathrm{T}_{\mathrm{s}}-1+\mathrm{T}_{\mathrm{h}}-1$

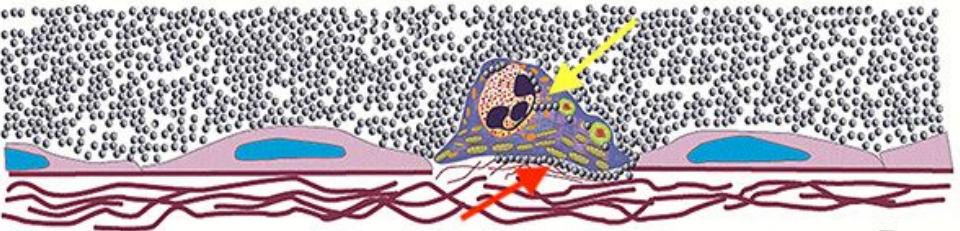

$\mathrm{T}_{\mathrm{s}}-2+\mathrm{T}_{\mathrm{h}}-2$
A

B

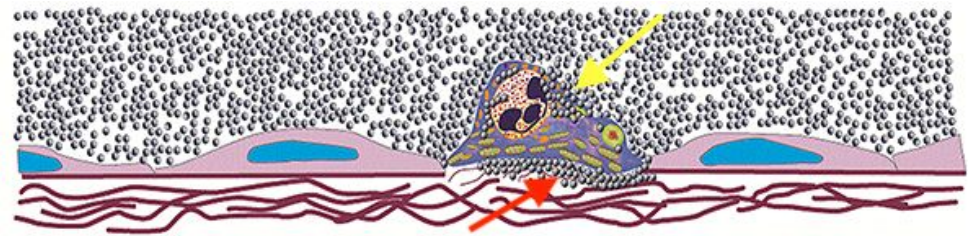

$\mathrm{T}_{\mathrm{s}-} 3+\mathrm{T}_{\mathrm{h}-} 3$

C

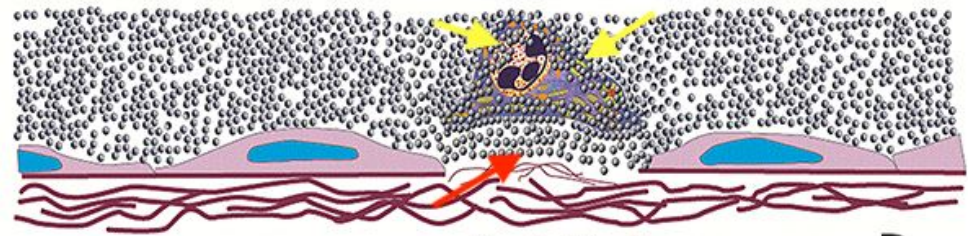

$\mathrm{T}_{\mathrm{s}}-4+\mathrm{T}_{\mathrm{h}-}-4$

\section{Figure 1}

The scoring standard of contrast-enhanced ultrasound scoring system. A: Ts 1: no or less than 1/2 of the substrate could be seen with the mixing of spot-like ( $<3 \mathrm{~mm}$ ) contrast agent (red arrow); Th 1: no contrast agent was found on the surface of the thrombus. B: Ts 2: more than 1/2 of the substrate could be seen with the mixing of crack-like contrast agent (red arrow); Th 2: a small amount of dot-like contrast agent was seen on the surface of the thrombus (yellow arrow). C: Ts 3: a wide gap (>3 $\mathrm{mm}$ ) of ultrasound contrast agent could be seen in most of the substrate (red arrow); Th 3: strip-like contrast agent was seen on the surface of the thrombus (yellow arrow). D: Ts 4: no or narrow/thin substrate (red arrow); Th 4: a large amount of the contrast agent entered the surface of the thrombus (yellow arrow). 

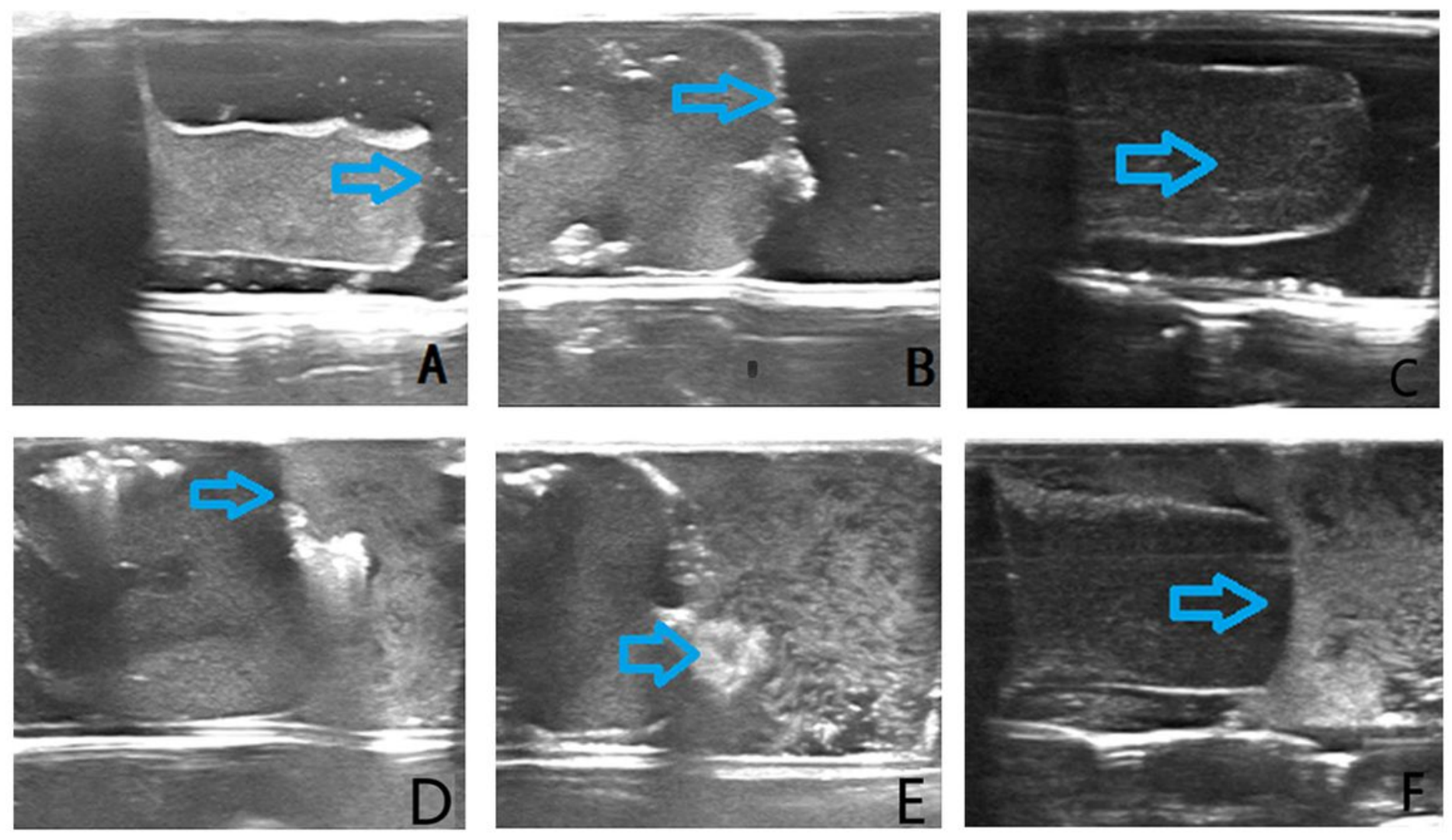

\section{Figure 2}

The two-dimensional and contrast-enhanced ultrasound appearance of the thrombus at 1 hour, 2 hours and 7 days. A: One hour after thrombus formation, the surface of the thrombus was hypoechoic (blue arrow). B: Two hours after the formation of the thrombus, the volume of the thrombus increased, the echo of the surface of the thrombus was enhanced and it was hyperechoic (blue arrow). C: Seven days after the thrombus formed, the inside of the thrombus was uniformly hypoechoic, and the surface echo was slightly stronger (blue arrow). D: One hour after thrombus formation, the ultrasound contrast agent can enter the freshly formed part of the thrombus surface (blue arrow), which makes the contrast morphology inconsistent with the 2-dimensional morphology. E: Two hours after the formation of the thrombus, a small thrombus (blue arrow) with obvious mobility on the surface of the thrombus can be seen, which is not shown in the two-dimensional image. F: No ultrasound contrast agent entered the surface of the thrombus at 7 days (blue arrow). 


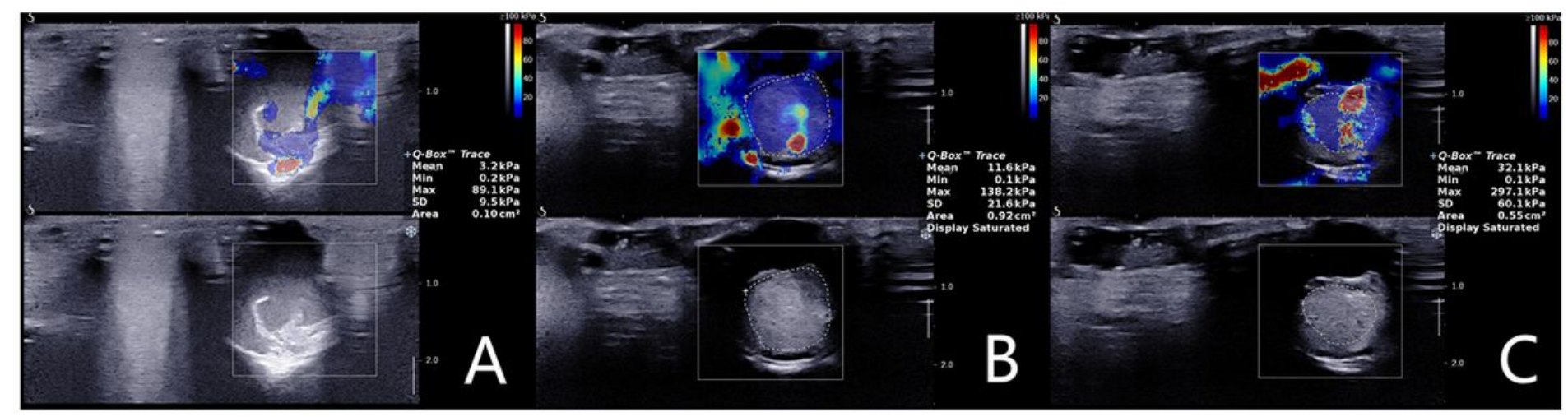

\section{Figure 3}

Shear wave elastography images at different time periods after thrombosis. A: fresh thrombosis; B: thrombus formed within 1 hour; C: thrombus formed over 7 days.

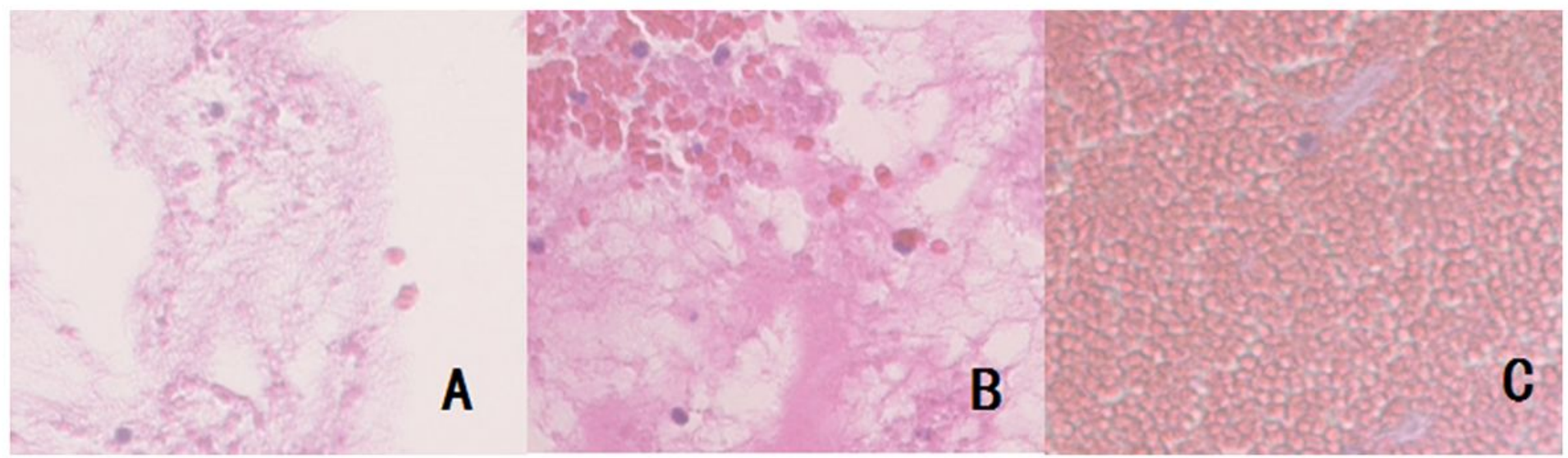

\section{Figure 4}

Pathologic staining results (Hematoxylin-eosin staining $\times 200$ ). A: The newly formed thrombi had the loosest texture and minimal cell aggregation. B: The fibrous network structure was loose and the cell space was large shows that 1 hour after thrombus formation. C: The structure of the red blood cells and fiber network was uniform, and the intercellular space was small on the seventh day. 


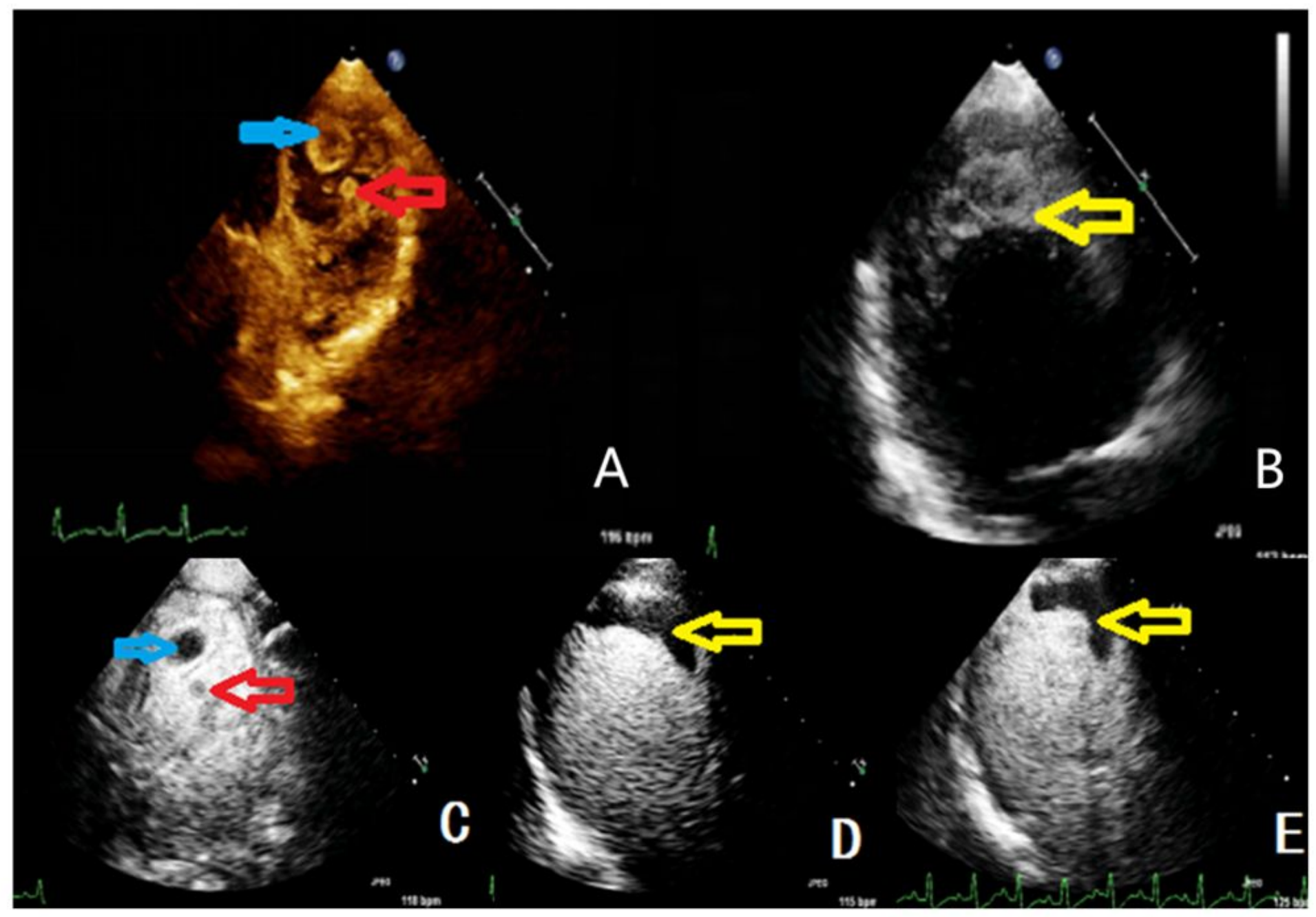

Figure 5

A woman aged 18 years with infective endocarditis of 11 years' duration was diagnosed with cerebral, renal, and lower extremity arterial embolism. TTE and CEUS showed three thrombi in the left ventricular cavity. All of the thrombi resolved after thrombolytic therapy. Figure A: TTE showed two solid, quasicircular thrombi (thrombus 1 - blue arrow, thrombus 2 - red arrow) in the left ventricle. Figure B: TTE showed one solid, irregular thrombus (thrombus 3 - yellow arrow) in the left ventricle apex. Figures $\mathrm{C}$ : The CEUS showed a large amount of contrast agent entered the around of the thrombus 1 (blue arrow) and thrombus 2 (red arrow). The morphology was inconsistent with the 2-dimensional morphology. There was no substrate noted in thrombus 1 and thrombus 2, the CEUS score was Ts $4+$ Th 4. Figures D: The CEUS showed a large amount of contrast agent entered the around of the thrombus 3 (yellow arrow). Wide gap accumulation of contrast agent can be seen in most of the base of the thrombus 3 , the CEUS score was Ts $3+$ Th 4. Figures E: The CEUS showed that the contrast agent entered the thrombus 3 (yellow arrow) in the form of spots after 9 days of thrombolytic treatment. The CEUS score was Ts $3+$ Th 2 . 